\title{
A COMMUTATIVE AND COMPACT DERIVATIONS FOR W* ALGEBRAS
}

\author{
ABDELGABAR ADAM HASSAN ${ }^{1,2, *}$, MOHAMMAD JAWED ${ }^{1}$ \\ ${ }^{1}$ Jouf University, College of Science and Arts in Tabrjal, Department of Mathematics, Kingdom of Saudi \\ Arabia \\ 2 University of Nyala, Department of Mathematics, Sudan \\ *Corresponding Author: aahassan@ju.edu.sa
}

ABSTRACT. In this paper, we study the compact derivations on $\mathrm{W}^{*}$ algebras. Let $\mathrm{M}$ be $\mathrm{W}^{*}$-algebra, let $\mathrm{LS}(\mathrm{M})$ be algebra of all measurable operators with $\mathbf{M}$, it is show that the results in the maximum set of orthogonal predictions. We have found that $\mathrm{W}^{*}$ algebra $A$ contains the Center of a $\mathrm{W}^{*}$ algebra $\mathrm{B}$ and is either a commutative operation or properly infinite. We have considered derivations from $\mathrm{W}^{*}$ algebra two-sided ideals.

\section{INTRODUCTION}

Let $M$ be a $\mathrm{W}^{*}$-algebra and let $Z(M)$ be the center of $M$. Fix $a \in M$ and consider the inner derivation $\delta_{a}$ on $M$ generated by the component $a$, which is $\delta_{a}(\cdot):=[a, \cdot]$.

The norm closing two sided ideal $f(B)$ generated by the finite projections of a $\mathrm{W}^{*}$ algebra $B$ behaves somewhat similar to the idealized compact operators of $B(H)$ (see [11],[8],[9]). Therefore, it is natural to ask about any sub-algebras $d$ of $B$ that is any derivation from A into $f(B)$ implemented from an element of $y(B)$.

Received April 1st, 2020; accepted April 20th, 2020; published May 28th, 2020.

2010 Mathematics Subject Classification. 47L15.

Key words and phrases. commutative; compact; operation; $\mathrm{W}^{*}$-algebras.

(C)2020 Authors retain the copyrights of their papers, and all open access articles are distributed under the terms of the Creative Commons Attribution License. 
We perform two main difficulties: the presence of the center of $B$ and the fact that the main characteristic in [8] proof (that is, if $Q_{n}$, is a sequence of mutually orthogonal projections and $T \in B(H)$ hence $\left\|Q_{n} T Q_{n}\right\|>\alpha>0$ for all $n$ implies that $T$ is not compact) failure to generalize to the case in which $g$ is of Type $I I_{\infty}$.

Finally, we have considered derivations from $d$ at the two-sided $C_{1+\varepsilon}(B, \tau)=B \cap L^{1+\varepsilon}(B, \tau)(1 \leq 1+\varepsilon<\infty)$ to obtain faithful finite normal trace $\tau$ on $B$.

\section{NOTATIONS PRELIMINARY}

Lemma (1). Let $B$ be a semi-finite algebra, let $Q_{0} \in p(B)$ and $x_{0} \in Q_{0}$ be such that $\omega_{x_{0}}$, is a faithful trace on $B_{Q_{0}}$. Assume there are $Q_{n} \in p(B), F_{n} \in p(\ell)$ and $U_{n} \in B$ for $n=n_{i}, \mathrm{n}_{i+1}, \ldots$, such that the projections $Q_{n}$ are mutually orthogonal and $Q_{n}=U_{n} U_{n}^{*}, Q_{o} F_{n}=U_{n}^{*} U_{n}$ for all $n$ (i.e., $\left.Q_{n} \sim Q_{0} F_{n}\right)$. Let $x_{n}=U_{n} F_{n} x_{0}$. Then $x_{n} \rightarrow_{J R W} O$.

Proof. Assume that $\sum_{n=n_{i}}^{\infty} Q_{n}=n_{i}$. Let $\tau$ be a faithful semi-finite normal $(f s n)$ trace on $B^{+}$to be agreed on $B_{Q_{0}}$ with $\omega_{x_{0}}$. Then for all $B \in B_{Q_{n}}^{+}$we have

$$
\begin{aligned}
\tau(B) & =\tau\left(U_{n} U_{n}^{*} B U_{n} U_{n}^{*}\right) \\
& =\tau\left(U_{n}^{*} U_{n} U_{n}^{*} B U_{n}\right) \\
& =\tau\left(Q_{o} F_{n} U_{n}^{*} B U_{n} F_{n} Q_{o}\right) \\
& =\omega_{x_{0}}\left(F_{n} U_{n}^{*} B U_{n} F_{n}\right) \\
& =\omega_{x_{n}}(B) .
\end{aligned}
$$

Let $P \in p(B)$ be any semi-finite projection. Then by [11] there is a central decomposition of the identity $\sum_{\gamma \in \Gamma} E_{\gamma}=1, E_{\gamma} \in p(\ell), E_{\gamma} E_{\gamma^{\prime}}=0$ for $\gamma \neq \gamma^{\prime}$ such that $\tau\left(P E_{\gamma}\right)<\infty$ for all $\gamma \in \Gamma$. Then

$$
\begin{aligned}
\tau\left(P E_{\gamma}\right)= & \sum_{n=1}^{\infty} \tau\left(Q_{n} P E_{\gamma} Q_{n}\right) \\
= & \sum_{n=1}^{\infty} \omega_{x_{n}}\left(Q_{n} P E_{\gamma} Q_{n}\right) \\
& \sum_{n=1}^{\infty}\left\|P E_{\gamma} x_{n}\right\|^{2}<\infty
\end{aligned}
$$


whence $\left\|P E_{\gamma} x_{n}\right\|<0$ for all $\gamma \in \Gamma$. Let $\varepsilon>0$ and let $\Lambda \subset \Gamma$ be a finite index set such that $\sum_{\gamma \notin \Lambda}\left\|E_{\gamma} x_{0}\right\|^{2}<\varepsilon$. Then for all $n$,

$$
\begin{aligned}
\sum_{\gamma \notin \Lambda}\left\|P E_{\gamma} x_{n}\right\|^{2} & =\sum_{\gamma \notin \Lambda}\left\|P E_{\gamma} U_{n} F_{n} x_{0}\right\|^{2} \\
& =\sum_{\gamma \notin \Lambda}\left\|P U_{n} F_{n} E_{\gamma} x_{0}\right\|^{2} \\
& \leq \sum_{\gamma \notin \Lambda}\left\|E_{\gamma} x_{0}\right\|^{2}<\varepsilon
\end{aligned}
$$

Hence from $\left\|P x_{n}\right\|^{2} \leq \sum_{\gamma \in \Lambda}\left\|P E_{\gamma} x_{n}\right\|^{2}+\varepsilon$ where $\left\|P x_{n}\right\| \rightarrow 0$, to completes the proof.

Lemma (2). Let $T \notin f(P)$, then there is an $\alpha>0$ and $0 \neq E \in p(\ell)$ such that for every $0 \neq F \in p(\ell)$ with $F \leq E$ we have $\|\pi(T F)\|>\alpha$.

Proof. Let $\alpha=\frac{1}{2}\|\pi(T)\| \neq 0$ and let $G$ be the sum of a maximal family of mutually orthogonal central projections $G_{\gamma}$ such that $\left\|\pi\left(T G_{\gamma}\right)\right\| \leq \alpha$. Then $\|\pi(T G)\|=\sup _{\gamma}\left\|\pi\left(T G_{\gamma}\right)\right\| \leq \alpha$, hence $G \neq 1$. Let $E=Z-G$ and let $0 \neq F \in P(\ell)$ with $F \leq E$. Since $F G=0$, by the maximally of the family we have $\|\pi(T F)\|>\alpha$.

\section{Relatively COMPACT DeRIVATION}

Let $M$ be a $W^{*}$-algebra and let $Z(M)$ be the center of $M$. Fix $a \in M$ and consider the inner derivation $\delta_{a}$ on $M$ generated by the element $a$, that is $\delta_{a}(\cdot):=[a, \cdot]$. Obviously, $\delta_{a}$ there is a linear bounded operator on $\left(M,\|\cdot\|_{M}\right)$, where $\|\cdot\|_{M}$ is a $C^{*}$-norm on $M$. It is known that there exists $c \in Z(M)$ such that the following estimate holds: $\left\|\delta_{a}\right\| \geq\|a-c\|_{M}$. In view of this result, it is natural to ask whether there exists is an element $y \in M$ with $\|y\| \leq 1$ and $c \in Z(M)$ such that $|[a, y]| \geq|a-c|$.

Definition (3). A linear subspace $I$ in the $\mathrm{W}^{*}$ algebra $M$ equipped with a norm $\|\cdot\|_{I}$ is said to be a symmetric operator ideal if 
(i) $\|S\|_{I} \geq\|S\|$ for all $S \in I$,

(ii) $\left\|S^{*}\right\|_{I}=\|S\|_{I}$ for all $S \in I$,

(iii) $\|A S B\|_{I} \leq\|A\|\|S\|_{I}\|B\|$ for all $S \in I, A, B \in M$.

Observe, that every symmetric operator ideal $I$ is a two-sided ideal in $M$, and therefore by [13], it follows from $0 \leq S \leq T$ and $T \in I$ that $S \in I$ and $\|S\|_{I} \leq\|T\|_{I}$.

Corollary (4). Let $M$ be a $W^{*}$-algebra and let $I$ be an ideal in $M$. Let $\delta: M \rightarrow I$ be a derivation. Then there exists an element $a \in I$, such that $\delta=\delta_{a}=[a, \cdot]$.

Proof. Since $\delta$ is a derivation on a $W^{*}$-algebra, it is necessarily inner [8]. Thus, there exists an element $d \in M$, such that $\delta(\cdot)=\delta d(\cdot)=[d, \cdot]$. It follows from the hypothesis that $[d, M] \subseteq I$.

Using [22] (or [20]), we obtain $\left[d^{*}, M\right]=-[d, M]^{*} \subseteq I^{*}=I$ and $\left[d_{k}, M\right] \subseteq I, k=1,2$, where $d=d_{1}+i d_{2}, d k=d_{k}^{*} \in M$, for $k=1,2$. It follows now, that there exist $c_{1}, c_{2} \in Z(M)$ and $u_{1}, u_{2} \in U(M)$, such that $\left|\left[d_{k}, u_{k}\right]\right| \geq 1 / 2\left|d_{k}-c_{k}\right|$ for $k=1,2$. Again applying [20], we obtain $d_{k}-c_{k} \in I$, for $k=1,2$. Setting $a:=\left(d_{1}-c_{1}\right)+i\left(d_{2}-c_{2}\right)$, we deduce that $a \in I$ and $\delta=[a, \cdot]$.

Corollary (5). Let $M$ be a semi-finite $\mathrm{W}^{*}$-algebra and let $E$ be a symmetric operator space. Fix $a=a^{*} \in S(M)$ and consider inner derivation $\delta=\delta_{a}$ on the algebra $L S(M)$ given by $\delta(x)=[a, x], x \in L S(M)$. If $\delta(M) \subseteq E$, then there exists $d \in E$ satisfying the inequality $\|d\|_{E} \leq\|\delta\|_{M \rightarrow E}$ and such that $\delta(x)=[d, x]$.

Proof. The existence of $d \in E$ such that $\delta(x)=[d, x]$. Now, if $u \in U(M)$, then $\|\delta(u)\|_{E}=\|d u-u d\|_{E} \leq\|d u\|_{E}+\|u d\|_{E}=2\|d\|_{E}$. Hence, if $\quad x \in M_{1}=\{x \in M:\|x\| \leq 1\}$, then $x=\sum_{i=1}^{4} \alpha_{i} u_{i}, \quad$ where $\quad u_{i} \in U(M) \quad$ and $\quad\left|\alpha_{i}\right| \leq 1 \quad$ for $\quad i=1,2,3,4, \quad$ and $\quad$ so $\|\delta(x)\|_{E} \leq \sum_{i=1}^{4}\left\|\delta\left(\alpha_{i} u_{i}\right)\right\|_{E} \leq 8\|d\|_{E}$, that is $\|\delta\|_{M \rightarrow E} \leq 8\|d\|_{E}<\infty$. 


\section{A COMMUtative Operation ON W* Sub-Algebras}

When $A$ a commutative operation is is crucial because it provides the following explicit way to find an operator $T \in B$ implementing the derivation.

For the rest of this section let $\mathrm{A}$ be any a commutative operation sub-algebras of $B$ and $\delta: \mathrm{A} \rightarrow B$ be any derivation. Let $u$ be the unitary group of $\mathrm{A}$ and $M$ be a given invariant mean on $u$, i.e., a linear functional on the algebra of bounded complex-valued functions on $u$ such that

(i) For all real $f, \inf \{f(U) \mid U \in u\} \leq M f \leq \sup \{f(U) \mid U \in u\}$

(ii) For all $U \in u, M f_{U}=M S$, where $f_{U}(V)=f(U V)$ for $V \in u$.

Thus $M$ is bounded and $|M f| \leq \sup \{|\mathrm{f}(U)| \mid \mathrm{U} \in \mathrm{u}\}$ for all $\mathrm{f}$ (see [8] for the existence and properties of $M)$.

For each $\phi \in B_{*}$ the map

$$
\phi \rightarrow M \phi\left(U^{*} \delta(U)\right)
$$

is linear and bounded and hence defines an element $T \in\left(B_{*}\right)^{*}$. Explicitly,

$$
\phi(T) \rightarrow M \phi\left(U^{*} \delta(U)\right) \quad \text { for all } \phi \in B_{*}
$$

The same easy computation as in [8] shows that $\delta=a \mathrm{~A} T$. Notice that for all $A \in B$ the map

$$
\phi \rightarrow M \phi\left(U^{*} B U\right)=\phi(E(B))
$$

defines an element $E(B)$ which clearly belongs to $A \cap B$. Moreover it is easy to see that $E$ is a conditional expectation (i.e., a projection of norm one) from $B$ onto $A \cap B$ (see [6]).

Theorem (6). Let $\mathrm{A}$ be a commutative operation $\mathrm{W}^{*}$ sub-algebras of $B$ containing the center $\ell$ of $B$. For every derivation $\delta: \mathrm{A} \rightarrow f(B)$ there is a $T \in f(B)$ such that $\delta=a \mathrm{~A} T$.

We have seen that given an invariant mean $M$ on $u$ there is a unique $T \in B$ such that $\delta=a \mathrm{~A} T$ and $E(T)=0$. We are going to show that $T \in \mathrm{A}(B)$. Reasoning by contradiction assume that $T \notin \mathrm{A}(B)$. We proof requires several reductions to the restricted derivation 
$\delta_{E}: \mathrm{A}_{E} \rightarrow f(B)$ for some $0 \neq E \in p(\ell)$. To simplify notations we shall assume each time that $E=1$

Let us start by noticing that if $Q_{i} \in p(\mathrm{~A})$ for $i=n, n+1, Q_{n}, Q_{n+1}=0$ and $P=Q_{n}+Q_{n+1}$, then

$$
P T P=\sum_{i=n}^{n+1} Q_{i} T Q_{i}+\delta\left(Q_{n+1}\right) Q_{n}+\delta\left(Q_{n}\right) Q_{n+1}
$$

hence

$$
\|\pi(P T P)\|=\left\|\sum_{i=n}^{n+1} \pi\left(Q_{i} T Q_{i}\right)\right\|+\max _{i} \pi\left(Q_{i} T Q_{i}\right)
$$

Definition (7). For every $Q \in p(\mathrm{~A})$ define $[Q]=[Q, \varepsilon]$ to be the central projection. Set

$$
P=\{P \in p(\mathrm{~A}) \mid[P]=1\} \text {. }
$$

Thus $P \in p$ iff $\|\pi(P T P G)\|=\|\pi(T G)\|$ for all $G \in p(\ell)$. We collect several properties of $[Q]$.

Corollary (8). Let $B$ be a semi-finite $\mathrm{W}^{*}$ algebra with a trace $\tau$, let $\mathrm{A}$ be a properly infinite $\mathrm{W}^{*}$ sub-algebras of $B$ and let $1 \leq 1+\varepsilon<\infty$. Then for every derivation $\delta: \mathrm{A} \rightarrow C_{1+\varepsilon}(B, \tau)$ there is $a T \in C_{1+\varepsilon}(B, \tau)$ such that $\delta=a \mathrm{~A} T$.

In the notations introduced there, it is easy to see that $\left.\phi\left(C_{1+\varepsilon}(B, \tau)\right)=C_{1+\varepsilon}(\tilde{B}, \tilde{\tau})\right)$, where $\tau=\tau \oplus \tau_{0}$ and $\tau_{0}$ is the usual trace on $B\left(H_{0}\right)$. We can actually simplify the proof by choosing $\tilde{\mathrm{A}}_{n}=I \otimes \ell$ since the condition $\ell \subset \mathrm{A}$ is no longer required.

Corollary (9). Let $P=Q_{n}+Q_{n+1}$. Then there is a largest central projection $\left[Q_{n}, Q_{n+1}\right]$ such that for every $G \in p(\ell)$ with $G \leq\left[Q_{n}, Q_{n+1}\right]$, we have $\left\|\pi\left(Q_{1} T Q_{1} G\right)\right\|=\|\pi(P T P G)\|$.

Proof. Let $G_{i}=\left\{G \in p(\ell) \mid\left\|\pi\left(Q_{i} T Q_{i} G\right)\right\|=\|\pi(P T P G)\|\right\}$ and $\Xi=\{G+\varepsilon \in p(\ell) \mid$ if $G \in p(\ell)$ and $\varepsilon \geq 0$ then $\left.G \in G_{n}\right\}$. Since $\|\pi(P T P G)\|=\max _{i}\left\|\pi\left(Q_{i} T Q_{i} G\right)\right\|$ for all $G \in p(\ell)$, we see that $G_{n} \cup G_{n+1}=p(\ell)$. Notice that $\Xi$ is hereditary (i.e., $G-\varepsilon \in \Xi$ and $F \in p(\ell), \mathrm{F} \leq G+\varepsilon$ imply $F \in \Xi)$. 
Let $\left[Q_{n}, Q_{n+1}\right]=\sup \Xi$. We have only to show that $\left[Q_{n}, Q_{n+1}\right] \in \Xi$. Let $G+\varepsilon=\sum_{\gamma}(G+\varepsilon)_{\gamma}$ be the sum of a maximal collection of mutually orthogonal projections $(G+\varepsilon)_{\gamma} \in \Xi$. Then for every $F \in \Xi$ we have $\left(\left[Q_{n}, Q_{n+1}\right]-(G+\varepsilon)\right) F=0$ because of the maximal of the collection of $\Xi$. Then $\left[Q_{n}, Q_{n+1}\right]=G+\varepsilon$. Consider now any $G \in p(\ell), \varepsilon \geq 0$, then $G=\sum_{\gamma} G(G+\varepsilon)_{\gamma}$ and since $G(G+\varepsilon)_{\gamma} \leq(G+\varepsilon)_{\gamma} \in \Xi$, we have $\left\|\pi\left(Q_{n} T Q_{n} G(G+\varepsilon)_{\gamma}\right)\right\|=\left\|\pi\left(P T P G(G+\varepsilon)_{\gamma}\right)\right\|$ for all $\gamma$. Since $\pi\left(Q_{n} T Q_{n} G\right)($ resp. $\pi(P T P G))$ is the direct sum of then

$$
\begin{aligned}
& \pi\left(Q_{n} T Q_{n} G(G+\varepsilon)_{\gamma}\right)\left(\operatorname{resp} . \pi\left(P T P G(G+\varepsilon)_{\gamma}\right)\right), \text { then we have } \\
& \qquad \begin{aligned}
\left\|\pi\left(Q_{n} T Q_{n} G\right)\right\| & =\sup _{\gamma}\left\|\pi\left(Q_{n} T Q_{n} G(G+\varepsilon)_{\gamma}\right)\right\| \\
& =\sup _{\gamma}\left\|\pi\left(P T P G(G+\varepsilon)_{\gamma}\right)\right\| \\
& =\|\pi(P T P G)\|
\end{aligned}
\end{aligned}
$$

whence $G \in G_{n}$. Since $\varepsilon \geq 0$ is arbitrary, we have $G+\varepsilon=\left[Q_{n}, Q_{n+1}\right] \in \Xi$ which completes the proof.

Corollary (10). (i) If $Q_{n} Q_{n+1}=0$ with $Q_{i} \in p(\mathrm{~A})$ then $1-\left[Q_{n}, Q_{n+1}\right] \leq\left[Q_{n}, Q_{n+1}\right]$.

(ii) If $Q_{n} \leq Q_{n+1}$ with $Q_{i} \in p(\mathrm{~A})$ then $\left[Q_{n}\right] \leq\left[Q_{n+1}\right]$.

(iii) If $\varepsilon \geq 0$ with $Q \in p(\mathrm{~A}), Q+\varepsilon \in p$ then $[Q]=[Q, \varepsilon]$ and $1-[Q] \leq[\varepsilon]$

If $\pi(T G) \neq 0$ for all $0 \neq E \in p(\ell)$ then the following hold:

(iv) If $E \in p(\ell)$ then $E=[E]$.

(v) If $Q \in p(\mathrm{~A})$ then $[Q] \leq c(Q)$, where $c(Q)$ is the central support of $Q$.

Proof. We have to show that for every $G \in p(\ell), G \leq 1-\left[Q_{n}, Q_{n+1}\right]$ we have $G \in G_{n+1}$. Let $E+\varepsilon$ be the sum $\sum_{\gamma} E_{\gamma}$ of a maximal collection of mutually orthogonal projections of $G_{n+1}$ that are majored by $G$. Then 


$$
\begin{aligned}
\left\|\pi\left(Q_{n} T Q_{n} F\right)\right\| & =\sup _{\gamma}\left\|\pi\left(Q_{n} T Q_{n} F_{\gamma}\right)\right\| \\
& =\sup _{\gamma}\left\|\pi\left(Q_{n+1}+Q_{n+1}\right) T\left(Q_{n+1}+Q_{n+1}\right) F_{\gamma}\right\| \\
& =\left\|\pi\left(Q_{n+1}+Q_{n+1}\right) T\left(Q_{n+1}+Q_{n+1}\right) F\right\|
\end{aligned}
$$

whence $E+\varepsilon \in G_{n+1}$. By the maximalist of the collection, $0 \leq G-(E+\varepsilon)$ does not majority any nonzero projection of $G_{n+1}$ and since $p(\ell)=G_{n} \cup G_{n+1}$, any central projection $G^{\prime} \leq G-(E+\varepsilon)$ must be in $G_{n}$. By definition of $\Xi$, this implies that $G-(E+\varepsilon) \in \Xi$ whence $G-(E+\varepsilon) \leq\left[Q_{n}, Q_{n+1}\right]$. So, $G-(E+\varepsilon) \leq G \leq 1-\left[Q_{n}, Q_{n+1}\right]$ and hence $G=E+\varepsilon \in G_{n+1}$ which completes the proof.

(ii) Let $G \in p(\ell)$ and $G \leq\left[Q_{n}\right]$. Then $\|\pi(T G)\|=\left\|\pi\left(Q_{n} T Q_{n} G\right)\right\| \leq\left\|\pi\left(Q_{n+1} T Q_{n+1} G\right)\right\|$ $\leq\|\pi(T G)\|$ whence equality holds and $\left[Q_{n}\right] \leq\left[Q_{n+1}\right]$ by the maximalist of $\left[Q_{n+1}\right]$.

(iii) $[Q, \varepsilon]$ is maximal under the condition: if $G \in p(\ell)$ and $G \leq[Q, \varepsilon]$ then

$$
\|\pi(Q T Q G)\| \leq\|\pi((Q+\varepsilon) T(Q+\varepsilon) G)\|=\|\pi(T G)\|
$$

which is the same condition defining $[Q, I-Q]=[Q]$. Thus $[Q]=[Q, \varepsilon]$. Applying this to $\varepsilon$ we have $[\varepsilon]=[\varepsilon, Q]$ and thus by (i) we have $[\varepsilon] \geq 1-[Q, \varepsilon]=1-[Q]$.

(ii) Let $E+\varepsilon, E \in p(\ell)$ then $\|\pi(E T E(E+\varepsilon))\|=\|\pi(T E(E+\varepsilon))\|$. This implies that if $\varepsilon \geq 0$, then $E+\varepsilon \leq[E]$ so $E \leq[E]$ and if $E+\varepsilon=[E]-E \leq[E]$ then $0=\|\pi(E T E(E+\varepsilon))\|=\|\pi(T(E+\varepsilon))\|$ whence $E=[E]$.

(v) Follows at once from (ii) and (iv).

The condition that $\|\pi(T E)\| \neq 0$ for all $0 \neq E \in p(\ell)$ is of course meaningless unless $B$ is properly infinite. Hence, we may assume without loss of generality that:

$B$ is properly infinite and semi-finite.

There is an $\alpha>0$ such that $\|\pi(T E)\|>\alpha$ for all $0 \neq E \in p(\ell)$. 
Lemma (11). Let $P \in p$ and $R_{n}=X_{P T P}[\alpha, \infty), R_{n+1}=X_{P T P}(-\infty,-\alpha]$, where $X_{P T P}()$ denotes the spectral measure of the self-adjoint operator $P T P$. Then there is an $E_{n} \in p(\ell)$, with $E_{n}=I-E$ such that $R_{i} E_{i}$ are properly infinite and $c\left(R_{i} E_{i}\right)=E_{j}$ for $i=n, \mathrm{n}+1$.

Proof. Let $R=R_{n}+R_{n+1}=X_{|P T P|}[\infty, \alpha)$ and let $F \neq 0$ be any central projection. If $R F$ were finite, we would have

$$
\begin{aligned}
\|\pi(T F)\| & =\|\pi(P T P F)\| \\
& =\|\pi(P T P(1-R) F)\| \\
& =\|\pi(|P T P|(1-R) F)\| \\
& \leq \alpha
\end{aligned}
$$

Thus $R F$ is infinite and nonzero. Hence $R$ is properly infinite and $c(R)=n$. Now let $E_{1}$ be the maximal central projection majored by $c\left(R_{n}\right)$, such that $R_{n} F_{n}$ is properly infinite. Then $c\left(R_{n}, E_{n}\right)=E_{n}$ and $R_{n}\left(n-E_{n}\right)$ is finite, hence $R_{n+}\left(n-E_{n}\right)=R_{n+1} E_{n+1}$ is properly infinite and $c\left(R_{n+1}, E_{n+1}\right)=E_{n+1}$.

End of the Proof of Theorem (6). Take any $0 \neq Q_{0} \in p(B)$ such that $B_{Q_{0}}$ has a faithful trace $\omega_{x_{0}}$ with $x_{0} \in Q_{0} H$ and assume $\left\|x_{0}\right\|=1$. Let $P_{\gamma} \in p, \gamma \in \Gamma$ be the not decreasing to zero. We are going to construct inductively a sequence $\gamma_{n} \in \Gamma, F_{n} \in p(\ell), Q_{n} \in p(B), U_{n}$ partial isometrics in $\mathrm{B}, x_{n} \in H$ such that

(a) $U_{n} U_{n}^{*}=Q_{n}, U_{n}^{*} U_{n}=Q_{0} F_{n}$, i.e., $Q_{n} \sim Q_{0} F_{n}$

(b) $x_{n}=U_{n} F_{n} x_{0} \in Q_{n} H$

(c) $Q_{n} Q_{m}=0$ for $n \neq m$

(d) $\gamma_{n}>\gamma_{m}\left(\right.$ hence $\left.P_{\gamma_{n}}<P_{\gamma_{m}}\right)$ for $n>m$

(e) $Q_{n} \leq p_{\gamma_{n}}$

(f) $\left\|p_{\gamma_{n+1}} x_{n}\right\|<1 / n$

(g) $\left|T x_{n}, x_{n}\right| \geq \alpha / 2$. 
The induction can be started with an arbitrary $P_{\gamma}$; assume we have the construction for $n-1$. Let us apply Lemma(11) to $P=P_{\gamma_{n}}$ and obtain $E_{i} \in p(\ell), R_{i} \in p(B)$ for $i=n, n+1$ as defined there. Then

$$
1=\left\|x_{o}\right\|^{2}=\left\|E_{n} x_{0}\right\|^{2}+\left\|E_{n+1} x_{0}\right\|^{2}
$$

Let $F_{n}$ be (any of) the projection $E_{n}$ or $E_{n+1}$ for which $\left\|E_{i} x_{0}\right\|^{2} \geq \frac{1}{2}$ and let $i$ be the corresponding index. Then $R_{i} F_{n}$ is properly infinite and has central support $F_{n}$. Now $Q_{0}$ is finite having a finite faithful trace $\omega_{x_{0}}$, hence so is $Q_{j} \sim F_{j} Q_{0} \leq Q_{0}$ for $1 \leq j \leq n-1$ and $\left(\sum_{j=1}^{n-1} Q_{j}\right) F_{n}$. Let $S_{n}=\inf \left\{R_{i} F_{n},\left(1-\sum_{j=1}^{n-1} Q_{j}\right) F_{n}\right\}$. By the parallelogram law (see [2]) applied to $F_{n}$ we have that

$$
R_{i} F_{n}-S_{n} \sim\left(\sum_{j=1}^{n-1} Q_{j}\right) F_{n}-\inf \left\{\left(\sum_{j=1}^{n-1} Q_{j}\right) F_{n},\left(1-R_{i}\right) F_{n}\right\}
$$

whence $R_{i} F_{n}-S_{n}$ is finite and hence $S_{n}$ is properly infinite and $c\left(S_{n}\right)=F_{n}$. Since $Q_{0} F_{n}$ is finite and $c\left(Q_{0} F_{n}\right) \leq F_{n}$ we have $Q_{0} F_{n} \prec S_{n}$, i.e., there is a partial isometry $U_{n} \in B$ and a $Q_{n} \in p(B), Q_{n} \leq S_{n}$ such that (a) holds. Let $x_{n}$ be defined by (b) and choose $\gamma_{n+1} \succ \gamma_{n}$ so that (d) and (f) hold. -Since $Q_{n} \leq R_{i} \leq P_{\gamma_{n}}$ we have (e), since $Q_{n} \leq\left(1-\sum_{j=1}^{n-1} Q_{j}\right) F_{n}$ we have (c). Finally $x_{n}=R_{i} x_{n}=P_{\gamma_{n}} x_{n}$ hence $(\mathrm{g})$ follows from

$$
\begin{aligned}
\left|\left(T x_{n}, x_{n}\right)\right| & =\left|\left(P_{\gamma_{n}} T P_{\gamma_{n}} x_{n}, x_{n}\right)\right| \\
& =\left|\left(P_{\gamma_{n}} T P_{\gamma_{n}} R_{i} x_{n}, R_{i} x_{n}\right)\right| \\
& \geq \alpha\left|\left(R_{i} x_{n}, R_{i} x_{n}\right)\right| \\
& =\alpha\left\|x_{n}\right\|^{2} \\
& =\alpha\left\|F_{n} x_{0}\right\|^{2} \\
& \geq \frac{1}{2} \alpha .
\end{aligned}
$$

Let now $y_{n}=x_{n}-P_{\gamma_{n+1}} x_{n}$. B is semi-finite, hence we can apply Lemma (1) to obtain that $x_{n} \rightarrow{ }_{B E W} 0$. Since $\left\|P_{\gamma_{n+1}} x_{n}\right\| \rightarrow 0$ we thus have $y_{n} \rightarrow{ }_{B E W} 0$ and $y_{n} \in P_{n} H$, where 
$P_{n}=P_{\gamma_{n}}-P_{\gamma_{n+1}} \in p(d)$ and are mutually orthogonal by (d). Clearly for $n$ large enough, $\left|\left(T y_{n}, y_{n}\right)\right|=\left|\omega_{y_{n}}(T)\right|>\frac{1}{4} \alpha$. Since $\omega_{y_{n}}(T)=M \omega_{y_{n}}\left(U^{*} \delta(U)\right)$, by the properties of the invariant mean mentioned, we have that $\sup \left\{\left|\omega_{y_{n}}\left(U^{*} \delta(U)\right)\right| \mid U \in u\right\}>\frac{1}{4} \alpha$. Thus we can find for every $n$, a unitary $V_{n} \in u$ such that $\left|\left(V_{n}^{*} \delta\left(V_{n}\right) y_{n}, y_{n}\right)\right|>\frac{1}{4} \alpha$. Let $A=\sum_{n=1}^{\infty} V_{n} P_{n}$, then $A \in d$ and

$$
\begin{aligned}
A^{*} \delta(A) y_{n}, y_{n} & =\left|\left(P_{n} A^{*} \delta(A) P_{n} y_{n}, y_{n}\right)\right| \\
& =\left|\left(P_{n}\left(A^{*} A T-A^{*} T A\right) P_{n} y_{n}, y_{n}\right)\right| \\
& =\left|\left(P_{n} V_{n}^{*} \delta\left(V_{n}\right) P_{n} y_{n}, y_{n}\right)\right| \\
& =\left|\left(V_{n}^{*} \delta\left(V_{n}\right) y_{n}, y_{n}\right)\right| \\
& =\frac{1}{4} \alpha
\end{aligned}
$$

for all $n$. Therefore $\left\|\delta(A) y_{n}\right\| \rightarrow 0$. But because of $(\Pi)$, we have $\delta(A) \notin f(B)$, which completes the proof.

\section{The Property of INFINITE $W^{*}$ SUb-ALGEbRA}

Lemma (12). Let $0<b \in Z(M), s(b)=1 ; e_{z}^{a}(0, \infty)$ be a properly infinite projection and $c\left(e_{z}^{a}(0, \infty)\right)=1$. Let projection $q \in P(M)$ be finite or properly infinite, $c(q)=1$ and $q \prec \prec e_{z}^{a}(0, \infty)$. Let $\mathbb{R} \ni \mu_{n} \downarrow 0$. For every $n \in \mathbb{N}$ we denote by $z_{n}$ such a projection that $1-z_{n}$ is the largest central projection, for which $\left(1-z_{n}\right) q \geq\left(1-z_{n}\right) e_{z}^{a}\left(\mu_{n} b,+\infty\right)$ holds. We have $z_{n} \uparrow_{n} 1$ and for

$$
d:=\left[\mu_{1} z_{1}+\sum_{n=1}^{\infty} \mu_{n+1}\left(z_{n+1}-z_{n}\right)\right] b
$$

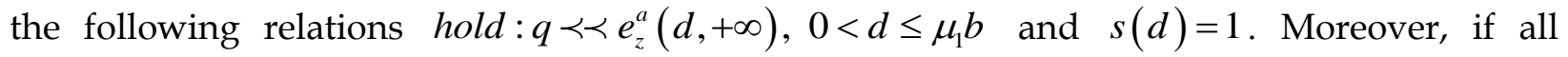
projections $e_{z}^{a}\left(\mu_{n} b,+\infty\right), n \geq 1$ are finite then $e_{z}^{a}(d,+\infty)$ is a finite projection as well.

Proof. Since, $e_{z}^{a}\left(\mu_{n+1} b,+\infty\right) \geq e_{z}^{a}\left(\mu_{n} b,+\infty\right)$ we have 
$e_{z}^{a}\left(1-z_{n+1}\right) q \geq\left(1-z_{n+1}\right) e_{z}^{a}\left(\mu_{n+1} b,+\infty\right) \geq\left(1-z_{n+1}\right) e_{z}^{a}\left(\mu_{n} b,+\infty\right)$. Hence, $z_{n+1} \geq z_{n}$ for every $n \in \mathbb{N}$. In addition, $e_{z}^{a}\left(\mu_{n} b,+\infty\right) \uparrow_{n} e_{z}^{a}(0,+\infty)$ and $e_{z}^{a}(0,+\infty)$ is properly infinite projection. Hence, in the case when $q$ is finite projection, it follows that $z_{n} \uparrow_{n} 1$. Let us consider the case when $q$ is a properly infinite projection with $c(q)=1$ and such that $q \prec \prec e_{z}^{a}(0, \infty)$. In this case, with $p=q, q=e_{z}^{a}(0,+\infty), q_{n}=e_{z}^{a}\left(\mu_{n} b,+\infty\right)$ and deduce $\vee_{n=1}^{\infty} z_{n} \geq c(q)=1$

All other statements follow from the form of element $d$. Since, $z_{1} d=\mu_{1} z_{1} b$, $\left(z_{n+1}-z_{n}\right)=\mu_{n+1}\left(z_{n+1}-z_{n}\right) b$ and $z_{n} q \prec \prec z_{n} e_{z}^{a}\left(\mu_{n} b,+\infty\right)$ for every $n \in \mathbb{N}$. Observe also that $s(d)=s(b)\left(z_{1}+\sum_{n=1}^{\infty}\left(z_{n+1}-z_{n}\right)\right)=1$.

Finally, let all projections $e_{z}^{a}\left(\mu_{n} b,+\infty\right), n \geq 1$ be finite. Since

$$
\begin{aligned}
d z_{1}=\mu_{1} b, d\left(z_{n+1}-z_{n}\right)= & \mu_{n+1} b\left(z_{n+1}-z_{n}\right), \text { we have } \\
e_{z}^{a}(d,+\infty) z_{1} & =e_{z}^{a}\left(\mu_{1} b,+\infty\right) z_{1}, \\
& e_{z}^{a}(d,+\infty)\left(z_{n+1}-z_{n}\right)=e_{z}^{a}\left(\mu_{n+1} b,+\infty\right)\left(z_{n+1}-z_{n}\right)
\end{aligned}
$$

for every $n \in \mathbb{N}$. There projections standing on the right-hand sides are finite. Hence, $e_{z}^{a}(d,+\infty)$ is finite projection as a sum of the left-hand sides [22].

We shall use a following well-known implication

$$
p \prec \prec q \Rightarrow z p \prec \prec z q, \quad \forall z \in P(Z(M)), 0<z \leq c(p) \vee c(q) .
$$

We supply here a straightforward argument. Let $z^{\prime} \in z \in Z(M)$ be such that $0<z^{\prime} \leq c(p z) \vee c(q z) z(c(p) \vee c(q))$. Then $z^{\prime} \leq c(p) \vee c(q)$ and therefore $z^{\prime}(z p)=z^{\prime} p \prec z^{\prime} q=z^{\prime}(z q)$. This means $z p \prec \prec z q$.

As in [6] we can use Theorem (6) to extend the result to the properly infinite case.

Theorem (13). Let A be a properly infinite $\mathrm{W}^{*}$ sub-algebra of $B$ containing the center $\ell$ of $B$. For every derivation $\delta: \mathrm{A} \rightarrow f(B)$ there is aT $\in f(B)$ such that $\delta=a \mathrm{~A} T$.

Before we start the proof let us recall that if $\mathrm{A}$ is properly infinite there is an infinite countable decomposition of the identity into mutually orthogonal projections of $\mathrm{A}$, all 
equivalent in $\mathrm{A}$ to $\mathrm{I}$, and thus a fortify equivalent in $B$ to 1 [8]. Therefore there is a spatial isomorphism

$$
\phi: B \rightarrow \tilde{B}=B \otimes B\left(H_{0}\right)
$$

with $H_{0}=l^{n+1}(\mathbb{Z})$ and

$$
\phi(\mathrm{A})=\tilde{\mathrm{A}}=\mathrm{A} \otimes B\left(H_{0}\right)
$$

[5]. Recall also that the elements $B$ of $\tilde{B}$ (or $\tilde{A}$ ) are represented by bounded matrices $\left[B_{i j}\right], i, j \in \mathbb{Z}$ with entries in $B$ (or A) by the formula

$$
\left(I \otimes E_{i j}\right) T\left(I \otimes E_{k l}\right)=T_{j k} \otimes E_{i l}
$$

where $E_{i j}$ is the canonical matrix unit of $B\left(H_{0}\right)$. In particular if $\ell, \wp$ are the maximal a commutative operation subalgebras of $B\left(H_{0}\right)$ of Laurent (resp. diagonal) matrices, then $B \in B \otimes \ell($ resp. $B \in B \otimes \wp)$ iff $\left[B_{i j}\right]$ is a Laurent matrix with entries in $B$, i.e., $B_{i j}=B_{i-j}$, where $B_{k}$, denotes the entry along the $k t h$ diagonal $\left(\operatorname{resp} . B_{i j}=\delta_{i j} B_{i i}\right)$ for all $i, j \in \mathbb{Z}$.

Proof. Let $\tilde{\delta}=\phi \circ \delta \circ \phi^{-1}$ then

$$
\tilde{\delta}: \tilde{d} \rightarrow \phi(f(B))=f(\tilde{B})
$$

is a relative compact derivation. Let us define the following $W^{*}$ algebras: $\tilde{\ell}=\tilde{B} \cap \tilde{B}, \tilde{\mathrm{A}}_{n}=\ell \otimes \ell, \mathrm{A}_{n}=\phi^{-1}\left(\tilde{\mathrm{A}}_{n}\right), \tilde{\mathrm{A}}_{n+1}=\mathrm{A} \otimes \ell$, and $\tilde{\mathrm{A}}_{n+2}=\mathrm{A}_{n} \otimes \wp$. First, let us notice that

$$
\begin{aligned}
\tilde{\mathrm{A}}_{n} \cap f(\tilde{B}) & =\left(\ell^{\prime} \otimes \ell\right) \cap\left(B \otimes B\left(H_{0}\right)\right) \cap f(\tilde{B}) \\
& =(B \otimes \ell) \cap f(\tilde{B}) \\
& =\{0\}
\end{aligned}
$$

by [22]. Therefore

$$
\mathrm{A}_{n}^{\prime} \cap f(B)=\phi^{-1}\left(\tilde{A}_{n}^{\prime}\right) \cap f(B)=\phi^{-1}\left(\tilde{\mathrm{A}}_{n}^{\prime} \cap f(\tilde{B})\right)=\{0\}
$$

because $\phi$ is spatial Now

$$
\begin{aligned}
\tilde{\ell} & =\left(B \otimes B\left(H_{0}\right)\right) \cap\left(B^{\prime} \otimes I\right) \\
& =\ell \otimes I \subset \tilde{\mathrm{A}}_{n} \subset \tilde{\mathrm{A}} .
\end{aligned}
$$


Thus we can apply Theorem(6) to the derivation $\tilde{\delta}$ restricted to the a commutative operation sub-algebra $\tilde{\mathrm{A}}_{n}$ of $\tilde{B}$ and we obtain a $T_{n} \in f(\tilde{B})$ such that $\tilde{\delta}_{n}=\tilde{\delta}-a \mathrm{~A} T_{n}$ vanishes on $\tilde{\mathrm{A}}_{n}$. Now

$$
\tilde{\mathrm{A}}_{n+1} \subset B \otimes \ell \subset \ell^{\prime} \otimes \ell=\tilde{\mathrm{A}}_{n}^{\prime}
$$

Therefore, for all $A_{n} \in \tilde{\mathrm{A}}_{n}$ and $A_{n+1} \in \tilde{\mathrm{A}}_{n+1}$ we have

$$
\tilde{\delta}_{n}\left(A_{n} A_{n+1}\right)=A_{n} \tilde{\delta}_{n}\left(A_{n+1}\right)=\tilde{\delta}_{n}\left(A_{n+1} A_{n}\right)=\tilde{\delta}_{n}\left(A_{n+1}\right) A_{n}
$$

i.e., $\tilde{\delta}_{n}\left(A_{n+1}\right)$ and $A_{n}$ commute and hence

$$
\tilde{\delta}_{n}\left(\tilde{\mathrm{A}}_{n+1}\right) \subset \tilde{\mathrm{A}}_{n}^{\prime} \cap f(\tilde{B})=\{0\}
$$

Thus $\tilde{\delta}_{n}$ also vanishes on $\tilde{\mathrm{A}}_{n+1}$. Now $\tilde{\mathrm{A}}_{n}$ is a commutative operation and hence so are $\mathrm{A}_{n}$ and $\tilde{\mathrm{A}}_{n+2}$. Moreover,

$$
\tilde{\ell} \subset \tilde{\mathrm{A}}_{n} \subset \tilde{\mathrm{A}} \subset \tilde{B}
$$

Implies

$$
\ell=\phi^{-1}(\tilde{\ell}) \subset \mathrm{A}_{n} \subset \mathrm{A} \subset B
$$

and hence

$$
\tilde{\ell}=\ell \otimes I \subset \mathrm{A}_{n} \otimes I \subset \tilde{\mathrm{A}}_{n+2} \subset \tilde{\mathrm{A}} \subset \tilde{B}
$$

Thus we can apply again Theorem(6) to the relative compact derivation $\tilde{\delta}_{n}$ restricted to $\tilde{\mathrm{A}}_{n+2}$. Let $T_{n+1} \in f(\tilde{B})$ be such that $\tilde{\delta}_{n}$ agrees with ad $T_{n+1}$ on $\tilde{\mathrm{A}}_{n+2}$. Since

$$
\mathrm{A}_{n} \otimes I \subset \mathrm{A} \otimes I \subset \mathrm{A} \otimes \ell=\tilde{\mathrm{A}}_{n+1}
$$

and $\tilde{\delta}_{n}$ vanishes on $\tilde{\mathrm{A}}_{n+1}$, we see that ad $T_{n+1}$ vanishes on $\mathrm{A}_{n} \otimes I$, i.e.,

$$
T_{n+1} \in\left(\mathrm{A}_{n} \otimes I\right)^{\prime} \cap f(\tilde{B})=\left(\mathrm{A}_{n}^{\prime} \otimes B\left(H_{0}\right)\right) \cap f g(\tilde{B})
$$

Then for all $i, j \in \mathbb{Z},\left(T_{n+1}\right)_{i j} \in \mathrm{A}_{n}^{\prime}$ and

$$
\left(T_{2}\right)_{i j} \otimes E_{n n}=\left(I \otimes E_{n i}\right) T_{n+1}\left(I \otimes E_{j n}\right) \in f(\tilde{B})
$$


whence by Lemma(12)(a) $\left(T_{n+1}\right)_{i j} \in f(B)$. But we saw that $d_{n}^{\prime} \cap f(B)=\{0\}$, hence $\left(T_{n+1}\right)_{i j}=0$ for all $i, j \in \mathbb{Z}$, so $T_{n+1}=0$. Therefore $\tilde{\delta}_{n}$ vanishes also on $\tilde{\mathrm{A}}_{n+2}$ and hence on $I \otimes \wp$. Now $\ell$ and $\wp$ generate $B\left(H_{0}\right)$, whence $\tilde{\mathrm{A}}_{n+1}=\mathrm{A} \otimes \ell$ and $I \otimes \wp$ generate $\tilde{\mathrm{A}}$. Thus by the $\sigma$-weak continuity of $\tilde{\delta}_{n}$ (see [6]) we see that

$\tilde{\delta}_{n}=\tilde{\delta}-a \mathrm{~A} T_{n}=0$, i.e., $\tilde{\delta}=a \mathrm{~A} T_{n}$. Clearly $\delta=a d \phi^{-1}\left(T_{n}\right)$ and $\phi^{-1}\left(T_{n}\right) \in \mathrm{A}(B)$.

Let us assume in this part that $B$ is semi-finite and let $\tau$ be a fsn trace on it. Beside the closed ideal $f(B)$ we can also consider the (non closed) two-sided norm-ideals $C_{1+\varepsilon}(B, \tau)$ for $1 \leq 1+\varepsilon<\infty$ defined by

$$
\begin{aligned}
C_{1+\varepsilon}(B, \tau) & =\left\{B \in B \mid \tau\left(|B|^{1+\varepsilon}\right)<\infty\right\} \\
\|B\|_{1+\varepsilon} & =\tau\left(|B|^{1+\varepsilon}\right)^{\frac{1}{1+\varepsilon}} \quad \text { for } B \in C_{1+\varepsilon}(B, \tau) .
\end{aligned}
$$

Obviously,

$$
C_{1+\varepsilon}(B, \tau)=B \cap L^{1+\varepsilon}(B, \tau),
$$

where the latter is the non commutative $L^{1+\varepsilon}$-space of $B$ relative to $\tau$ (see [14]).

Recall the following facts about $L^{1+\varepsilon}(M)$ spaces in the case of a general $\mathrm{W}^{*}$ algebra $M$ and $1 \leq 1+\varepsilon<\infty\left(L^{\infty}(M)\right.$ is identified with $\left.M\right): L^{1+\varepsilon}(M)$ is a Banach space, its dual is isomorphic to $L^{\frac{\varepsilon}{1+\varepsilon}}(M)$ (with $1 / 1+\varepsilon+{ }^{1+\varepsilon} / \varepsilon=1$ ), and the duality is established by the functional tr on $L^{1}(M)$, where if $\quad A \in L^{1+\varepsilon}(M), \quad B \in L^{\frac{\varepsilon}{1+\varepsilon}}(M) \quad$ we have $\quad A B, \quad B A \in L^{n}(M) \quad$ and

$$
\begin{aligned}
& \operatorname{tr}(A B)=\operatorname{tr}(B A),|\operatorname{tr}(A B)| \leq\|A\|_{1+\varepsilon}\|B\|_{\frac{\varepsilon}{1+\varepsilon}}, \\
&\|A\|_{1+\varepsilon}=\left(\operatorname{tr}|A|^{1+\varepsilon}\right)^{1 / 1+\varepsilon}=\max \left\{|\operatorname{tr} A B| \mid B \in L^{\frac{\varepsilon}{1+\varepsilon}}(M),\|B\|_{\frac{\varepsilon}{1+\varepsilon}} \leq 1\right\}
\end{aligned}
$$

(see [14]). Of course, if $M=B$ we can identify $L^{1+\varepsilon}(M)$ with $L^{1+\varepsilon}(B, \tau)$ and $\operatorname{tr}$ with $\tau$. The following inequality will be used here only in the semi-finite case and in the context of $C_{1+\varepsilon^{-}}$ ideals, but since the same proof holds for $L^{1+\varepsilon}$-spaces, we shall consider the general case. 
Corollary (14). Let $M$ be a $\mathrm{W}^{*}$ algebra, $\varepsilon \geq 0, A \in L^{1+\varepsilon}(M)$ and

$Q_{n}, Q_{n+1} \in p(M), Q_{n} Q_{n+1}=0, Q_{n}+Q_{n+1}=1$. Then

$$
\|A\|_{1+\varepsilon}^{1+\varepsilon} \geq\left\|Q_{n} A Q_{n}\right\|_{1+\varepsilon}^{1+\varepsilon}+\left\|Q_{n+1} A Q_{n+1}\right\|_{1+\varepsilon}^{1+\varepsilon}
$$

Proof. Let us first note that

$$
\left|\sum_{i=n}^{n+1} Q_{i} A Q_{i}\right|^{1+\varepsilon}=\sum_{i=n}^{n+1}\left|Q_{i} A Q_{i}\right|^{1+\varepsilon}
$$

And

$$
\left\|\sum_{i=n}^{n+1} Q_{i} A Q_{i}\right\|_{1+\varepsilon}^{1+\varepsilon}=\sum_{i=n}^{n+1}\left\|Q_{i} A Q_{i}\right\|_{1+\varepsilon}^{1+\varepsilon}
$$

Consider first $1+\varepsilon=n$ and take the polar decomposition's

$$
Q_{i} A Q_{i}=U_{i}\left|Q_{i} A Q_{i}\right|, i=n, n+1 .
$$

Then $U_{i} U_{i}^{*}$ and $U_{i}^{*} U_{i}$ are majored by $Q_{i}$ and hence $U_{i}$ commutes with $Q_{j}$. Therefore $B=\left(U_{n}+U_{n+1}\right)^{*}$ commutes with $Q_{i}$ and $\|B\|=1$. Then

$$
\begin{aligned}
\|A\|_{1} & \geq|\operatorname{tr} A B| \\
& =\left|\operatorname{tr}\left(\sum_{i=n}^{n+1} Q_{i} B A Q_{i}\right)\right| \\
& =\operatorname{tr}\left(\sum_{i=n}^{n+1} Q_{i} A Q_{i}\right) \\
& =\sum_{i=n}^{n+1}\left\|Q_{i} A Q_{i}\right\|_{n} .
\end{aligned}
$$

Consider now $\varepsilon>0$. Let $B \in L^{\frac{\varepsilon}{1+\varepsilon}}(M)$ be such that $\|B\|_{\frac{\varepsilon}{1+\varepsilon}} \leq 1$ and

$$
\left\|\sum_{i=n}^{n+1} Q_{i} A Q_{i}\right\|_{1+\varepsilon}=\operatorname{tr}\left(\left(\sum_{i=n}^{n+1} Q_{i} A Q_{i}\right) B\right) .
$$

Take the polar decomposition's $A=U|A|$ and $B=V|B|$, then $V U$ are in $M$ and $|A|,|B|$ are in $L^{1+\varepsilon}(M), L^{\frac{\varepsilon}{1+\varepsilon}}(M)$, respectively. Let 


$$
f(z)=\operatorname{tr}\left(\sum_{i=n}^{n+1} Q_{i} U|A|^{(1+\varepsilon) z} Q_{i} V|B|^{\left(\frac{\varepsilon}{1+\varepsilon}\right)(1-z)}\right)
$$

Then by standard arguments, it is easy to see that $f$ is analytic on $0<\operatorname{Re} z<n$ and continuous and bounded on $0 \leq \operatorname{Re} z \leq n$. Then by the three-line theorem (see [4]) we have

$$
f\left(\frac{1}{1+\varepsilon}\right) \leq \operatorname{Max}_{t \in \mathbb{R}} f(i t)^{\varepsilon / 1+\varepsilon} \operatorname{Max}_{t \in \mathbb{R}} f(1+i t)^{1 / 1+\varepsilon}
$$

Now $f\left(\frac{1}{1+\varepsilon}\right)=\left\|\sum_{i=n}^{n+1} Q_{i} A Q_{i}\right\|_{1+\varepsilon}$ and by Holder's inequality

$$
\begin{aligned}
& |f(i t)|=\operatorname{tr}\left|\left(\sum_{j=n}^{n+1} Q_{j} U|A|^{i(1+\varepsilon) t} Q_{j} V|B|^{-i\left(\frac{\varepsilon}{1+\varepsilon}\right) t}|B|^{\frac{\varepsilon}{1+\varepsilon}}\right)\right| \\
& \leq\left\|\sum_{j=n}^{n+1} Q_{j} U|A|^{i(1+\varepsilon) t} Q_{j} V|B|^{-i\left(\frac{\varepsilon}{1+\varepsilon}\right) t}\right\|\left\|\left|\left\|\left.\right|^{\frac{\varepsilon}{1+\varepsilon}}\right\|_{1}\right.\right. \\
& \left.\leq\left(\max _{j}\left\|Q_{j} U|A|^{i(1+\varepsilon) t} Q_{j}\right\|\right) \| V|B|^{-i\left(\frac{\varepsilon}{1+\varepsilon}\right)}\right)\|\| B \|_{\frac{\varepsilon}{1+\varepsilon}}^{\frac{\varepsilon}{1+\varepsilon}}
\end{aligned}
$$

$\leq n$.

Again by Holder's inequality applied twice and by the result already obtained in the $\varepsilon=0$ case,

$$
\begin{aligned}
|f(1+i t)| & =\operatorname{tr}\left|\left(\sum_{j=n}^{n+1} Q_{j} U|A|^{i(1+\varepsilon) t}|A|^{1+\varepsilon} Q_{j} V|B|^{-i\left(\frac{\varepsilon}{1+\varepsilon}\right) t}\right)\right| \\
& \leq\left\|\sum_{j=n}^{n+1} Q_{j} U|A|^{i(1+\varepsilon) t}|A|^{1+\varepsilon} Q_{j}\right\|\left\|V|B|^{-i\left(\frac{\varepsilon}{1+\varepsilon}\right) t}\right\| \\
& \leq\left\|U|A|^{i(1+\varepsilon) t}|A|^{1+\varepsilon}\right\|_{1} \\
& \leq\left\|U|A|^{i(1+\varepsilon) t}\right\|\left\|\left|\left\|\left.A\right|^{1+\varepsilon}\right\|_{1}\right.\right. \\
& \leq\|A\|_{1+\varepsilon}^{1+\varepsilon}
\end{aligned}
$$

Thus $f\left(\frac{1}{1+\varepsilon}\right) \leq\|A\|_{1+\varepsilon}$ whence by the second equality in this proof,

$$
\|A\|_{1+\varepsilon}^{1+\varepsilon} \geq\left\|\sum_{i=n}^{n+1} Q_{i} A Q_{i}\right\|_{1+\varepsilon}^{1+\varepsilon}=\sum_{i=n}^{n+1}\left\|Q_{i} A Q_{i}\right\|_{1+\varepsilon}^{1+\varepsilon}
$$

Data Availability

No data were used to support this study. 
Conflicts of Interest: The author(s) declare that there are no conflicts of interest regarding the publication of this paper.

\section{References}

[1] I. Chifan, S. Popa, J.O. Sizemore, Some OE- and $\mathrm{W}^{*}$-rigidity results for actions by wreath product groups, J. Funct. Anal. 263 (2012), 3422-3448

[2] M. Breijer, Fredholm theories in von algebras I, Math. Ann. 178 (1968), 243-254.

[3] E. Christensenex, Extension of derivations, J. Funct. Anal. 27 (1978), 234-247.

[4] J. Conway, Functions of One Complex Variable, 2nd ed., Springer- Verlag, New York,1978.

[5] J. Dixmier, Les Algebres d'operateurs dans 1'Espace Hilbertien, 2nd ed., Gauthier-Villars, Paris, 1969.

[6] F. Gilfeather and D. Larsinn, Nest-subalgebras of von Neumann algebras: commutants modulo compacts and distance estimates, J. Oper. Theory, 7 (1982), 279-302.

[7] A. Connes, E. Blanchard, Institut Henri Poincaré, Institut des hautes études scientifiques (Paris, France), Institut de mathématiques de Jussieu, eds., Quanta of maths: conference in honor of Alain Connes, non commutative geometry, Institut Henri Poincaré, Institut des hautes études scientifiques, Institut de mathématiques de Jussieu, Paris, France, March 29-April 6, 2007, American Mathematical Society ; Clay Mathematics Institute, Providence, R.I. : Cambridge, MA, 2010.

[8] S. Albeverio, Sh. Ayupov, K. Kudaybergenov, Structure of derivations on various algebras of measurable operators for type I von Neumann algebras, J. Funct. Anal. 256 (9) (2009), 2917-2943.

[9] V. Kaftal, Relative weak convergence in semifinite von Neumann algebras, Proc. Amer. Math. Soc. 84 (1982), 89-94.

[10]S. Sakal, $C^{*}$-Algebras and $W^{*}$-Algebras (Ergebnisse der Mathematik und ihrer Grenzgebiete, Vol. 60), Springer-Verlag, Berlin, New York, 1971.

[11] M. Takesaki, Theory of Operator Algebras I, Springer-Verlag, New York, 1979.

[12] N. Higson, E. Guentner, Group $C^{*}$-algebras and K-theory, in Noncommutative Geometry (Martina Franca, 2000), pp. 137-251. Lecture Notes in Math., 1831.

[13]D. Voiculescu, Free non-commutative random variables, random matrices and the $\mathrm{II}_{1}-$ factors of free groups, Quantum Probability and Related Topics VI, L. Accardi, ed., World Scientific, Singapore, 1991, pp. 473-487.

[14] A.F. Ber, F.A. Sukochev, Commutator estimates in $W^{*}$-factors, Trans. Amer.Math. Soc. 364(2012), 5571-5587.

[15]F. Murray, J. von Neumann: Rings of operators, IV, Ann. Math. 44(1943), 716-808. 
[16]J. Peterson, L2-rigidity in von Neumann algebras, Invent. Math. 175 (2009), 417-433.

[17] B.E. Johnson, S.K. Parrott, Operators commuting with a von Neumann algebra modulo the set of compact operators, J. Funct. Anal. 11 (1972), 39-61.

[18] R. Kadison, A note on derivations of operator algebras, Bull. Lond. Math. Soc. 7 (1975), 41-44.

[19]K. Dykema, Free products of hyperfinite von Neumann algebras and free dimension, Duke Math. J. 69 (1993), 97-119.

[20]C. Consani, M. Marcolli, Noncommutative geometry, dynamics, and $\infty$-adic Arakelov geometry, Selecta Math. 10 (2004), 167.

[21] A.F. Ber, F.A. Sukochev, Commutator estimates in W*algebras, J. Funct. Anal. 262 (2012), 537-568.

[22]D. Pask, A. Rennie, The noncommutative geometry of graph $\mathrm{C}^{*}$-algebras I: The index theorem, J. Funct. Anal. 233 (2006), 92-134.

[23]S. Popa, F. Radulescu, Derivations of von Neumann algebras into the compact ideal space of a semifinite algebra, Duke Math. J. 57(2)(1988), 485-518.

[24]I.E. Segal, A non-commutative extension of abstract integration, Ann. Math. 57 (1953), 401-457. 